\title{
Aspectos clave del diseño hidrológico de diques forestales en el arroyo de los Santos (Ávila)
}

\author{
Jiménez González, A. ${ }^{1}$, Diez Hernández, J.M..* \\ ${ }^{1}$ Trva/ Cinco Villas 10, $4^{\circ 2} .05003$ (Ávila). España \\ ${ }^{2}$ Universidad de Valladolid, Grupo de Ecohidrología Fluvial. Avda Madrid, 44.34004 (Palencia). España. \\ *e-mail: jmdiez@iaf.uva.es
}

\section{Resumen}

Una actuación típica en los proyectos de restauración hidrológico-forestal es el diseño de diques forestales. Como parte de la información de base para el dimensionamiento de este tipo de obras hidráulicas es preciso determinar la crecida de cálculo, la cual deberá ser evacuada ordenadamente por los dispositivos de desagüe que estén operativos en la estructuras. Durante este mismo evento de diseño, el proceso hidrológico de propagación de la crecida en la cubeta del dique hace que el dimensionamiento correcto del vertedero y los mechinales sea un aspecto relevante del diseño hidráulico de un dique.

Se han dimensionado dos diques forestales de retención y laminación de crecidas en el arroyo de los Santos (Ávila), atendiendo al comportamiento hidrológico más desfavorable esperado de la cuenca para la vida útil de los diques.

Mediante modelaje hidrológico con HEC-HMS se han obtenido los caudales de diseño, y modelado el tránsito de la crecida en las estructuras.

Palabras clave: corrección, HEC-HMS, Hidrotecnias, laminación, modelaje. 


\section{Introducción}

En la historia forestal española, los proyectos dedicados a la restauración han ocupado una parte importante desde la creación de la primera escuela de Ingenieros de Montes en 1846 (García López, 1995). Dentro de éstos los dedicados a la hidrología forestal han resultado de los más destacados. Es una actuación histórica que en sus bases poco ha cambiado a lo largo del tiempo. En cambio, en la actualidad el uso de la información y su procesamiento de manera rápida, nos permite obtener unos resultados eficaces con un mayor rendimiento. Esto no implica que sean sustitutivos de los métodos tradicionales.

En este trabajo se expone una metodología centrada en la determinación de los aspectos clave para un diseño de varias obras hidráulicas dentro de la misma cuenca. Se exponen los pasos clave para el diseño real y eficaz de acuerdo a la consecución de los objetivos establecidos.



Figura 1. Localización de la zona de actuación así como las diferentes decciones propuestas para la situación de los diques forestales en el arroyo de Los Santos. Fuente: Elaboración propia.

La zona de actuación se sitúa dentro del término municipal de El Herradón de Pinares, Ávila, Castilla y León (figura 1). Se caracteriza la zona por dar muestras de unos estados erosivos importantes, debido en gran medida a la ausencia de vegetación arbórea. Además ha sido incluida dentro del Plan Nacional de Actuaciones Prioritarias en materia de restauración hidrológico-forestal, control de la erosión y defensa contra la desertificación, como prioridad $2^{\mathrm{a}}$. La cuenca de estudio, está dentro de un espacio LIC y ZEPA, "Pinares del Bajo Alberche" y dentro del Monte de Utilidad Pública n69 "Dehesa boyal” (Junta de Castilla y León, 2003). 
El curso que discurre por la cuenca es el arroyo de Los Santos, tributario del arroyo de la Gaznata, que a su vez atraviesa por la localidad de El Herradón. Como referencia histórica que muestra el estado de las cuencas de la zona, y en concreto de la de estudio, la riada acaecida en 1999 en la que el arroyo de la Gaznata afectó de manera severa la localidad antes mencionada (Ministerio del Interior, Dirección General de Protección Civil y de Emergencias, 2007).

Como se ha descrito, estamos ante una actuación típica en el medio natural en una comarca con tipología plenamente forestal, en la que como en gran parte de nuestro territorio, se presentan una serie de condicionantes: protección de espacios protegidos, degradación del territorio, aspectos culturales...que hacen que una actuación deba de ser eficazmente estudiada y diseñada.

\section{Material y métodos}

Los objetivos que se persiguen con la actuación son los siguientes:

- Evaluar los estados erosivos de la cuenca.

- Evaluar los riesgos hidrológicos a la población y sus bienes.

- Plantear medidas correctoras eficaces

Como ya se ha mencionado anteriormente, el diseño de diques forestales, ha variado poco a lo largo del tiempo. Pero en cambio tanto material como metodología han experimentado grandes cambios. Se ha pasado de tener una información muy limitada, a una excesivamente extensa (Reque y Pérez, 2011). Por otro lado también nos permite obtener una solución más precisa en el diseño.

Así pues el material empleado podría dividirse en tres grupos; uno de análisis en oficina, de manera genérica, mediante la visualización en SIG de ortofotos actuales e históricas, modelos del terreno, mapas de vegetación, edafológicos, geológicos y de otro tipo (Allué Andrade, 1990; Elena-Roselló et al., 1997; IGME, 2009; MAGRAMA, 2001, 2007a, 2007b, 2008, 2010; Rivas Martínez et al., 1987), otro de análisis específico en materia hidrológica como es el software HEC-HMS para modelaje, NumCur como apoyo para la definición de la escorrentía de la cuenca (Navarro Hevia, 2009), Retorno 2.0 para analizar la serie de datos y Bloques SWMM para generar una lluvia sintética, ambos desarrollados por la Universidad Politécnica de Barcelona. Por último otro grupo de material empleado en campo como la estación total para realizar el levantamiento topográfico así como el material complementario como prismas, o aquel de toma de muestras para apertura de calicatas y análisis del suelo. Es importante incidir en esta última parte, totalmente necesaria dado que en el medio natural el software debe ser de apoyo para el técnico.

Una vez definidos los materiales empleados, y los objetivos, se estructura una metodología que persigue la consecución de los objetivos expuestos. Dentro de la misma diferenciamos varias etapas, descritas en rasgos generales a continuación: 


\subsection{Caracterización de la cuenca}

Se trata de la parte más descriptiva, en la que se analiza la forma de la cuenca, el relieve, la red hidrográfica, la climatología, la edafología y geología y la fauna y flora.

Es un punto totalmente necesario para cualquier actuación en el medio natural y que no se diferenciará en gran medida para otros proyectos, salvo que se presta una atención mayor cómo es la red hidrográfica de cuenca y cómo condicionan los parámetros físicos de la misma a su funcionamiento. Para ello resulta de gran ayuda tanto las diferentes capas en SIG, pero además el análisis en campo de la cuenca. Además la consulta de trabajos previos como los de Antón Pacheco, 2009, Alonso et al., 2009, y Díez Herrero, 2001 resultan de gran utilidad.

\subsection{Análisis del funcionamiento hidrológico}

Como resultado del punto anterior se procede al análisis de los datos obtenidos. En concreto se aplican una serie de pasos. En primer lugar se evalúan los estados erosivos, diferenciando diferentes unidades de suelos, y mediante el método del número de curva se obtienen y unos valores que se ponderarán por superficie para obtener un valor general para la cuenca. En segundo lugar es de gran relevancia para el diseño de diques de laminación y retención, la emisión de sedimentos de la cuenca en toneladas/año y que condicionarán su funcionamiento útil (De Aranda et al., 1992). Pasados estos pasos, se procede a realizar un estudio de los datos estadísticos de pluviometría y que son aplicables para la zona (Martínez de Azagra y Navarro Hevia, 2007). Este paso tiene la finalidad de comprobar que dentro de la serie no hay ninguna anomalía destacable y por tanto es fiable. En cuarto lugar, se procede al cálculo de la precipitación máxima diaria $\left(\mathrm{P}_{\max }\right)$ para diferentes periodos de retorno $(\mathrm{T}=50 ; 100)$ y según las diferentes metodologías (Gumbel, DGC, LogPearson, SQRT-ET $\max$ ). Para ello se apoyará en software informático (Retorno 2.0) pero también se acepta válido el cálculo tradicional.

Elegido un periodo de retorno adecuado, se procede al cálculo de la escorrentía superficial y a su transformación en caudal. Para ello se proponen diferentes metodologías, algunos más teóricos como el método modificado de Témez y otros más prácticos como el método del Hidrograma Unitario Triangular (HUT) (Chow, 1994), que aplica el software HEC-HMS. Pero para ello se necesita conocer de qué manera se produce esta precipitación máxima. Es necesario generar una lluvia sintética basándose en el valor obtenido de Pmax (Dirección General de Carreteras, 1999). Se simulará una precipitación con diferentes distribuciones (general, general media y centrada). Una vez conocido el caudal emitido por la cuenca, es necesario especificarlo para las diferentes secciones de cierre en las que se situarán los diques. Por último es necesario conocer el volumen de agua embalsado en el caso más desfavorable dado que el comportamiento del dique que se situará aguas arriba influirá sobre el siguiente elemento aguas abajo. Conociendo la curva de almacenamiento-altura se aplicará la metodología "Puls" que relaciona mediante la 
ecuación de continuidad el caudal de entrada $\mathrm{I}(\mathrm{t})$ y de salida $\mathrm{Q}(\mathrm{t})$ con el almacenamiento $\mathrm{S}(\mathrm{t})$. Así este caudal de salida del primer dique (el que está aguas arriba), será el caudal de diseño.

\subsection{Diseño de las obras}

Obtenidos los caudales de diseño, se procede al dimensionamiento de las obras, para que cumplan su funcionamiento de manera eficaz y tratando de minimizar el impacto sobre el medio natural. Se diseñan por tanto, dos diques con contradique y cuenco de amortiguación tal y como se aprecia en la figura 2 y con las características de la tabla 1, apoyándose en el software propuesto por Martínez de Azagra, 1993. Además como se ha descrito en el anterior paso, se obtiene el almacenamiento del dique $\mathrm{n}^{\circ} 1$ (Tab. 2), para considerar la emisión de caudal para el segundo aguas abajo.

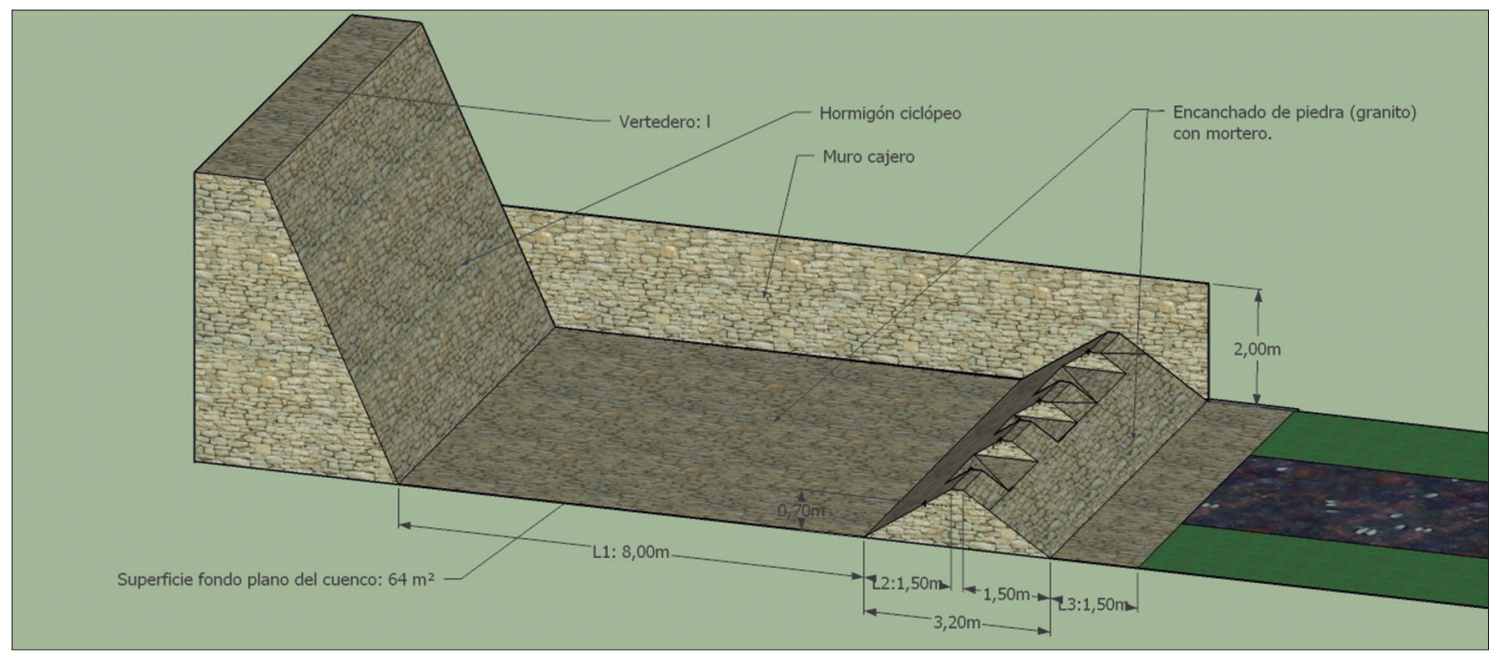

Figura 2. Croquis en $3 \mathrm{D}$ del cuenco amortiguador con contradique dentado y uno de los muros cajeros en un lateral. Fuente: Elaboración propia.

Tabla 1. Datos descriptivos de los dos diques propuestos.Fuente: Elaboración propia.

\begin{tabular}{|c|c|c|}
\hline & Dique $\mathbf{~ n}^{\mathbf{1}}$ & Dique n'2 \\
\hline Altura útil & $5 \mathrm{~m}$ & $3.5 \mathrm{~m}$ \\
\hline Espesor de la base & $3.5 \mathrm{~m}$ & $3 \mathrm{~m}$ \\
\hline Espesor en coronación & $1.2 \mathrm{~m}$ & $1 \mathrm{~m}$ \\
\hline Altura del vertedero & $1.5 \mathrm{~m}$ & $1.2 \mathrm{~m}$ \\
\hline Longitud del vertedero & $8 \mathrm{~m}$ & $7.5 \mathrm{~m}$ \\
\hline Caudal de cálculo (T $=100$ años) & $16.7 \mathrm{~m} 3 / \mathrm{s}$ & $15.4 \mathrm{~m} 3 / \mathrm{s}$ \\
\hline Capacidad máxima de vertido & $25.06 \mathrm{~m} 3 / \mathrm{s}$ & $16.8 \mathrm{~m} 3 / \mathrm{s}$ \\
\hline Número de Froude & 5.64 & 4.53 \\
\hline
\end{tabular}


Tabla 2. Relación cota almacenamiento del dique $\mathrm{n}^{\mathrm{0}} 1$. Correspondiente con el vaso del mismo. Datos obtenidos mediante levantamiento topográfico. Fuente: Elaboración propia.

\begin{tabular}{|c|c|}
\hline$H(m)$ & $S\left(\mathrm{~m}^{3}\right)$ \\
\hline 0 & 0 \\
\hline 1 & 13.36 \\
\hline 2 & 63.14 \\
\hline 3 & 319.46 \\
\hline 4 & 565.35 \\
\hline 5 & 806.43 \\
\hline
\end{tabular}

\section{Resultados}

Siguiendo con la anterior metodología, se evalúa y caracteriza la cuenca de manera que finalmente obtenemos unos valores que serán los de diseño de las obras. Aún así en el anterior apartado ya se han mostrado algunos resultados al aplicar dicha metodología. Además conviene destacar como más relevante los datos relativos a precipitaciones máximas diarias (Tab. 3) que servirán de base para la obtención de caudales (tabla 4). Posteriormente se harán los ajustes necesarios utilizando el software HEC-HMS como apoyo y viendo la laminación y el caudal de entrada y salida (Fig. 3):

Tabla 3. Precipitaciones máximas diarias (en mm) para periodos de retorno 50 y 100 años, según los diferentes métodos.

\begin{tabular}{|c|c|c|}
\hline & Pmaxd $_{\mathrm{T}=50}$ & Pmax $_{\mathrm{d}, \mathrm{T}=100}$ \\
\hline Gumbel (Teórica) & 111.06 & 124.42 \\
\hline Gumbel (Retorno 2.0) & 115.42 & 129.61 \\
\hline DGC (Dirección General de Carreteras) & 107.86 & 122.10 \\
\hline LogPearson III & 123.16 & 144.31 \\
\hline SQRT-ETmax & 111.69 & 129.61 \\
\hline Media & 113.84 & 130.01 \\
\hline
\end{tabular}

Tabla 4. Resumen de los caudales obtenidos por diferentes métodos para un periodo de retorno de 100 años.

\begin{tabular}{|c|c|}
\hline Método & $\boldsymbol{Q}_{\max 1 \mathrm{~T}}=100$ \\
\hline Modificadado de Témez & $18.53 \mathrm{~m}^{3} / \mathrm{s}$ \\
\hline HUT & -------------- \\
\hline - Distribución general & $16.80 \mathrm{~m}^{3} / \mathrm{s}$ \\
\hline - Distribución centrada & $17.40 \mathrm{~m}^{3} / \mathrm{s}$ \\
\hline - Distribución general media & $15.80 \mathrm{~m}^{3} / \mathrm{s}$ \\
\hline
\end{tabular}


Como ya se aprecia en la Tab. 4, los valores de caudales no difieren en gran medida entre sí por tanto se opta por una opción simple, a favor de la seguridad, aplicando el método modificado de Témez para el cálculo del caudal de diseño del dique ${ }^{\circ} 1$ (Tab. 1).

Aplicando la metodología Puls y conocida la relación cota-almacenamiento (Tab. 2), se puede obtener el comportamiento del dique $\mathrm{n}^{\circ} 1$ en la situación más desfavorable (Fig. 3). Lo cual nos permite obtener el caudal del diseño del dique $\mathrm{n}^{\circ} 2$, sin considerar aportes en la corta distancia que los separa (120 m aproximadamente).

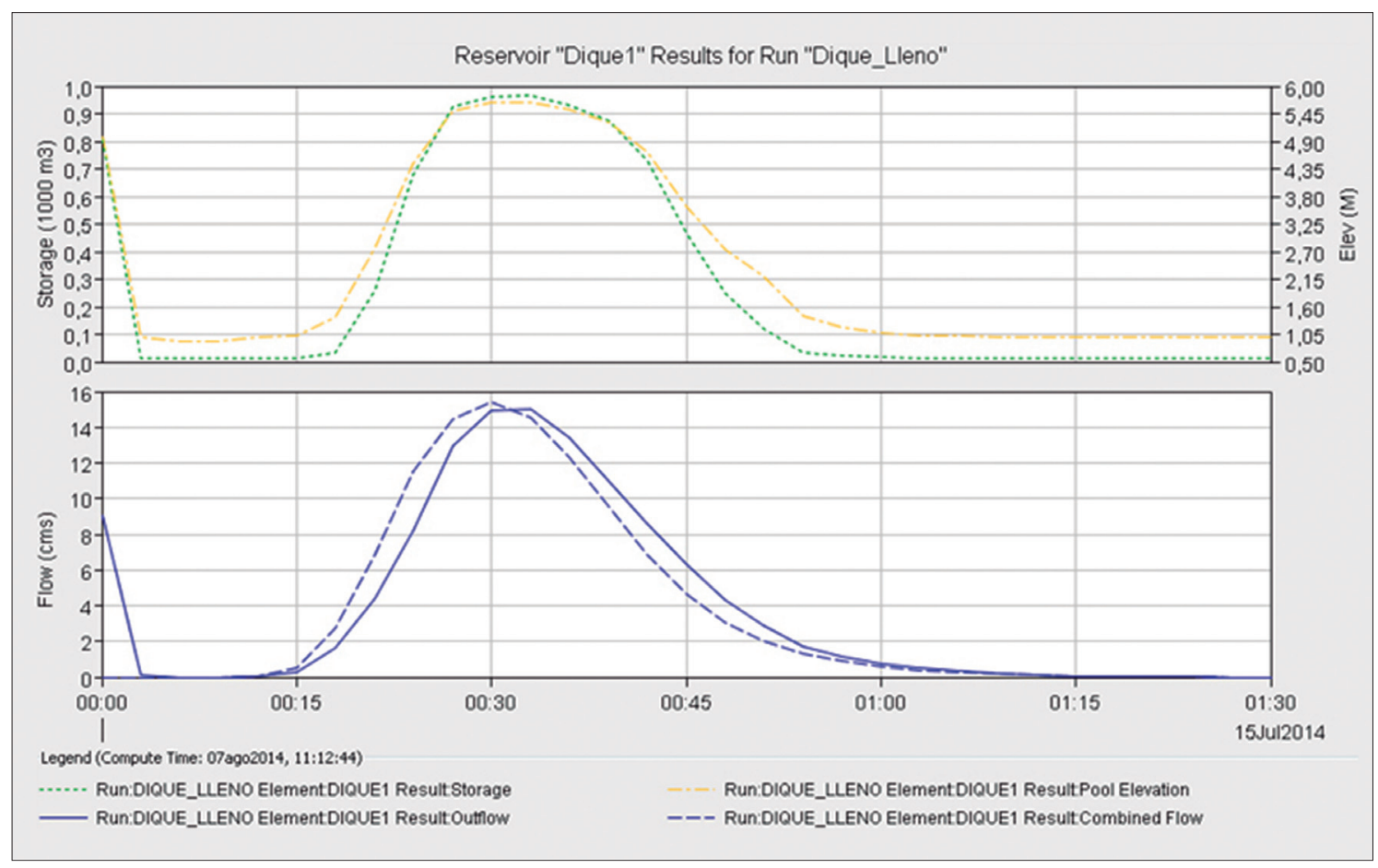

Figura 3. Hidrograma de salida del dique ${ }^{\circ} 11$ leno para el aguacero de cálculo máximo diario con periodo de retorno 100 años. Fuente: Elaboración propia.

El efecto laminador por tanto se debe de considerar para ambas obras. Teniendo en cuenta que el tiempo de concentración para la cuenca (a pie del dique $\mathrm{n}^{\circ} 2$ como sección de cierre), es de $29.61 \mathrm{~min}$, la acción de las estructuras diseñadas resulta eficaz. Esta eficacia se debe a que en su conjunto la distribución de caudales pico se retrasa (efecto laminador) hasta 1 hora aproximadamente, en la sección de cierre de la cuenca.

\section{Discusión}

Es de relevancia a la hora de diseñar cualquier estructura, considerar diferentes opciones y caminos. Desde un inicio se contemplan diferentes métodos para estimar la precipitación máxima diaria y se elige aquel que se considere el más cercano a la 
realidad (según diferentes informaciones). Con esta suposición y optando por aquella más segura y a la vez fiable, se consideran diferentes metodologías para el cálculo de caudales, volviendo de nuevo a tomar una nueva decisión. Dicha decisión se plasma en un resultado, el caudal de diseño de la obra. El mismo ha de influir tanto para el dique $n^{\circ} 1$ como para el $n^{\circ} 2$, dado la cercanía entre sí. En esa misma distancia no se consideran nuevas aportaciones dada la morfología de la cuenca en ese parte y los valores despreciables de la escorrentía en esa superficie respecto al caudal.

Se considera y evalúa una única opción, la más desfavorable (dique $\mathrm{n}^{\circ} 1,11$ eno), dado que será la más restrictiva y por tanto lo que se ha de tener en cuenta para el diseño. Se omiten las situaciones de dique vació u otras debido a que suponen un normal funcionamiento de la estructura hidráulica, y por tanto carecen de relevancia para dicho proceso de diseño. Hay que tener en cuenta que se pretende el diseño de manera eficaz y considerando el tiempo como un factor limitante, no es posible analizar todas las opciones pero si aquellas que pueden condicionar al resto.

\section{Conclusiones}

En rasgos generales, los aspectos fundamentales resaltados en esta metodología se consideran perfectamente válidos para cualquier lugar en el que se demande la realización de este tipo de obras de corrección hidrológica.

Como se ha visto, la acción de los diques supone una distribución de los caudales a lo largo del tiempo. La metodología aplicada para su diseño trata de ser lo más adecuada para el entorno considerando las características propias. Por otro lado las ligeras suposiciones y consideraciones que se toman a lo largo de dicho proceso, pueden alejar precisión a los resultados. Por eso es necesario seguir a lo largo del proceso un resguardo o margen de seguridad pero al mismo tiempo no caer en un error que pueda conllevar en un sobredimensionamiento.

Uno de los aspectos clave es la simulación tanto de la lluvia, acercándose a una situación más "real" o próxima a la realidad y por supuesto la simulación del comportamiento de dichas estructuras ante esta $\mathrm{P}_{\max }$ de extraordinaria ocurrencia.

Por otro lado, la efectividad de la obra para el entorno en el cual se encuadra (vegetación rala y puntual arbustiva), puede ser aumentada si se complementase el proyecto con diferentes biotecnias de restauración que permitan la instalación de una cubierta vegetal protectora.

Esto, definitiva, permite una optimización del diseño para una mayor eficacia sobre el medio natural y por supuesto, sobre la disminución de riesgos para los bienes y seres humanos.

\section{Bibliografía}

Allue Andrade, J.L.,1990. Atlas fitoclimático de España. Madrid: Ministerio de Agricultura Pesca y Alimentación: Instituto Nacional de Investigaciones Agrarias (INIA). 
Alonso, G., Blanco, D., Vives, J., 2009. Estudio de inundabilidad del río Gaznata a su paso por la localidad del Herradón, Ávila. Máster en Ingeniería y Gestión del Agua EOI Escuela de Negocios 2008-2009.

Antón-Pacheco Almodóvar, J., 2009. Proyecto básico de ubicación, diseño y construcción de diques para laminación de avenidas en la cabecera del río Gaznata. Restauración Hidrológico Forestal de la Cuenca. Universidad de Salamanca. Escuela Politécnica Superior de Ávila.

Chow, V.T., 1994. Hidrología aplicada. Bogotá, Colombia: McGraw-Hill

De Aranda, G. et al., 1992. Hidrología forestal y protección de suelos. Técnicas y experiencias en dirección de obras. Madrid: Organismo Autónomo Parques Nacionales.

Díez Herrero, A., 2001. Geomorfología e hidrología fluvial del río Alberche. Modelos y S.I.G. para la gestión de riberas. Memoria presentada para optar al grado de doctor. Madrid: Universidad Complutense de Madrid: Facultad de Ciencias Geológicas.

Dirección General de Carreteras. Ministerio de Fomento de España, 1999. Máximas lluvias diarias en la España Peninsular. Madrid: Ministerio de Fomento de España.

Elena-Roselló, R., et al., 1997. Clasificación biogeoclimática de España peninsular y balear. Madrid: Ministerio de Agricultura Pesca y Alimentación: Instituto Nacional de Investigaciones Agrarias (INIA).

García López, J.M., 1995. Breve repertorio histórico de los orígenes de la Ordenación de Montes en España (1852-1899). Cuadernos de la Sociedad Española de Ciencias Forestales, 1. 139-148. Madrid.

IGME, 2009. Mapa Geológico 1:50000. Hoja 532. Obtenido el 21 de julio de http://www. igme.es/internet/cartografia/cartografia/magna50.asp.

Junta de Castilla y León. (Ed.) (2003). Catálogo de los montes de utilidad pública de la Provincia de Ávila. Zamora: Ediciones Monte Casino (Benedictinas).

Magrama, 2001. Mapa plan de actuaciones prioritarias en materia de restauración hidrológica-forestal. Obtenido el 25 de julio de http://www.magrama.gob.es/es/desarrollorural/temas/politica-forestal/desertificacion-restauracion-forestal/restauracion-hidrologico-forestal/rhf_plan_restauracion.aspx.

Ministerio del Interior, Dirección General de Protección Civil y de Emergencias (2007). Catálogo Nacional de Inundaciones Históricas. Cuencas Tajo y Júcar. Fascículo 2. DVD. Versión 2.0.

Magrama, 2007a. Mapa zonas LIC en la provincia de Ávila. Obtenido el 15 de agosto de http://www.magrama.gob.es/es/biodiversidad/temas/espacios-protegidos/red-natura2000/lic_castillayleon.aspx.

Magrama, 2007b. Mapa zonas ZEPA en la provincia de Ávila. Obtenido el 15 de agosto de http:/www.magrama.gob.es/es/biodiversidad/temas/espacios-protegidos/red-natura2000/zepa_castillayleon.aspx\#para0.

Magrama, 2008. Mapa Plan Nacional de Acción Nacional contra la Desertificación. Obtenido el 25 de julio de http://www.magrama.gob.es/en/desarrollo-rural/temas/politica-forestal/desertificacion-restauracion-forestal/lucha-contra-la-desertificacion/lch_ pand_descargas.aspx.

Magrama, 2010. Mapa del número de incendios forestales en España durante el decenio 2001-2010. Obtenido el 25 de julio de http://www.magrama.gob.es/es/biodiversidad/estadisticas/.

Martínez de Azagra, A., 1993. Manual para el programa DIQ2.exe. Valladolid: Universidad 
de Valladolid. Escuela Técnica Superior de Ingenierías Agrarias (Palencia).

Martínez de Azagra, A.; Navarro Hevia, J., 2007. Hidrología Forestal. El ciclo hidrológico. Valladolid: Universidad de Valladolid, Secretariado de Publicaciones e Intercambio Editorial, 2009.

Navarro Hevia, J.; Martínez de Azagra, A., Mongil Manso, J., 2009. Hidrología de conservación de aguas: captación de precipitaciones horizontales y escorrentías en zonas secas. Valladolid: Universidad de Valladolid, Secretariado de Publicaciones e Intercambio Editorial, 2009.

Reque J.; Pérez R., 2011. Del Monte al Rodal. Manual SIG de Inventario Forestal. Valladolid: Universidad de Valladolid-Vicerrectorado de Docencia.

Rivas Martínez, S., et al., 1987. Memoria del mapa de series de vegetación de España. Madrid: ICONA. 